\title{
Similarity analysis of the streamer zone of Blue Jets
}

\author{
N.A. Popov ${ }^{1}$, M.N. Shneider ${ }^{2}$ and G. M. Milikh ${ }^{3}$
}

${ }^{1}$ Moscow State University, Moscow, Russia

${ }^{2}$ Department of mechanical and aerospace engineering Princeton University, Princeton, NJ, USA

${ }^{3}$ Astronomy Department University of Maryland, College Park, MD, USA

\begin{abstract}
Multiple observations of Blue Jets (BJ) and Gigantic Blue Jets (GBJ) show that BJ and GBJ emits a fan of streamers similar to a laboratory leader. Moreover, in the exponential atmosphere those long streamers grow preferentially upward, producing a narrow cone confined by the aperture angle. It was also noticed that BJ are similar to the streamer zone of a leader (streamer corona) and the modeling studies based on the streamers fractal structure were conducted. Objective of this paper is to study the fractal dimension of the bunch of streamer channels emitted by BJ and GBJ, at different altitude and under the varying electric field. This similarity analysis has been done in three steps: First we described the dendritic structure of streamers in corona discharge applying the fractal theory. Then using this model and the data from existing laboratory experiments we obtained the fractal dimension of the branching streamer channels. Finally the model was validated by the observations of BJ available from the literature.
\end{abstract}

\section{Introduction}

About two decades ago, researchers discovered upward-propagating collimated flashes of light originating above thunderstorms [Wescott et al., 1995]. Due to their distinctive, principally, blue color, they were termed "blue jets" (BJ). They resemble tall trees with quasi-vertical trunk and filamentary branches. [Pasko et al., 2002] discovered the so-called gigantic blue jets (GBJ), propagating into the lower ionosphere. Number of GBJ observations were made since [Su et al., 2003; Kuo et al., 2009; Cummer et al., 2009; Chou et al., 2011; Neubert et al., 2011; Soula et al. 2011; Liu et al., 2015].

Multiple observations of BJ show that they emits a streamers similar to the streamers of corona discharge of a laboratory leader. Streamers in corona discharge are self-similar plasma structures termed fractals [Wiesmann et al., 1986; Pasko et al., 2000; Pasko et al., 2002; Popov, 2002]. According to the current understanding the streamer propagates due to the formation of electron avalanches in the strong electric field $E$ of the space charge near the streamer tip. The propagation direction of those avalanches is determined by the shape of the streamer's electric field. The probability of streamer propagation in a given direction is determined by the spatial distribution of the electric field near the streamer tip. 
In the case of BJs the leader trunk barely exits the cloud and we only see the streamer zone [Raizer et al., 2006] and [da Silva and Pasko, 2013]. Contrary to BJs GBJs lightning leaders escape the thundercloud upwards and propagate through the stratosphere. An interesting hypothesis [da Silva and Pasko, 2013] stated that when GBJ reach the jump altitude when the streamer zone length is longer than the scale height of the atmospheric air density profile, the streamer zone reahes the ionosphere.

Note that we focus on study of the streamer corona, which is the basic of the blue jets (BJs), as well as gigantic blue jets (GBJs). Furthermore BJs and GBJs are of the similar structure, and the both are eventually evolve into the streamer region. Notice that in this paper we limit ourselves to the analysis of the structure of the streamer region of GBJ since it is much better recognizable on the existing images.

The dendritic structure of streamers in corona discharge was treated by means of the fractal theory [Satpathy, 1986], which main parameter is the fractal dimension $D$. Note that the fractal dimension $D$ of the plasma structure is determined by the power index $\gamma$, which depends on the reduced electric field value near the streamer tip [Popov, 2002]. [Pasko and George, 2002] numerically simulated the streamer corona of a positive leader as a fractal process. They suggested that the power index $\gamma$, that links the probabilities a random choice with electric field value, is $\gamma=1$ and obtained the fractal dimension of the dendritic structure $D=1.37 \pm 0.13$. The numerical simulations of the streamer corona of blue jets and blue starters as a fractal structures were first presented in [Pasko and George, 2002] although computed for $\gamma=1$.

The objective of this paper is to apply the theory of fractal dimension of streamers in the corona discharge to study formation and propagation of blue jets and gigantic blue jets. The proposed model concerns GJs, BJs and streamer corona. However, since the existing images of the streamer corona and of BJs cannot resolve the object's spatial structure, in the present paper we focus entirely on the GBJs. Thus the model is validated by comparison with the existing optical observations of gigantic blue jets.

\section{Fractal dimension of a bunch of multiple streamers}

68

Widely used model [Niemeyer et al., 1984; Wiesmann et al., 1986] describes the spatial structure of branching channels. In this model, the plasma channels propagate toward the highest electric field, provided that the field exceeds the threshold value $E_{c r}$. The spatial distribution of the electric field is determined from solution of the Laplas equation for the potential having the boundary conditions which consider the conducting channels. All these channels are taking as equal, while the field inside the channels is constant. The probability of the streamer propagation in $\mathrm{z}$-direction $\mathrm{P}_{\mathrm{z}}$ is determined by the field distribution near the streamer head. 
where $E_{z}$ is the field in the direction $\mathrm{z}, \gamma$ is the power index.

According to [Popov, 2002], the power index $\gamma$ can be obtained from the equation for the effective ionization frequency:

$\gamma=\frac{\partial \ln \left(v_{i o n}-v_{a t t}\right)}{\partial \ln E}$

where $v_{i o n}-v_{\text {att }}$ is the difference between the ionization frequency and electron attachment frequency for a given sort of the gas. It depends only on the reduced electric field $E / N$ value.

The major results of the model [Niemeyer et al., 1984; Wiesmann et al., 1986] is that the plasma structures allow spatial scaling, thus they are self-similar structures or fractals. As shown in [Satpathy, 1986] the fractal dimention $D$ depends entirely on the dimensionality of space $d$ and the power index $\gamma$ in Eq. (1), $D=D(d, \gamma)$.

Figure 1 reveals the power index as function of the reduced electric field $\gamma(E / N)$ for the air, here it is shown by the dashed line. It is computed for the $E / N$ range $200-1200 \mathrm{Td}$ ( 1 Townsend is equal to $\left.10^{-17} \mathrm{~V} / \mathrm{cm}^{2}\right)$. The respective ionization and electron attachment frequencies $v_{\text {ion }}(\mathrm{E} / \mathrm{N})$ and $v_{\text {att }}(\mathrm{E} / \mathrm{N})$ were obtained by the Bolsig+ code [Hagelaar and Pitchford, 2005].

In addition, Fig. 1 reveals the fractal dimension $D$ of the plasma structures formed in BJ, here it is shown by the solid line. The data $D(\gamma)=D(\gamma, d=3)$ obtained in [Satpathy, 1986] for the 3D Laplace field was used. These data were determined by the model [Niemeyer et al., 1984; Wiesmann et al., 1986] from the solution of 3-D Laplas equation for the potential having the boundary conditions which consider the conducting channels. [Satpathy, 1986] computed the number of conducting channels $N(R)$ which cross the sphere of radius $\mathrm{R}$. Then the fractal dimension $D(\gamma)$ was found using Eq. (5). The fractal dimension of the plasma structures vs. $E / N$ (Fig. 1) was found under the assumption that the maximum electric field in the streamer head is constant and equal to $E$.

The data [Kuo et al., 2009] shows the reduced electric field in the streamer head inside the GBJ in the altitude range $h=40-90 \mathrm{~km}$. It was obtained using the ratio of the observed intensities of the optical bands $\mathrm{N}_{2}(2 \mathrm{P})$ and $\mathrm{N}_{2}(1 \mathrm{~N})$. Found in the work [Kuo et al., 2009] maximum reduced electric field was $E / N=400 \mathrm{Td}$. Notice that due to the shift between the maximum electric field and maximum intensities of the $\mathrm{N}_{2}(2 \mathrm{P})$ and $\mathrm{N}_{2}(1 \mathrm{~N})$ bands in the streamer head [Naidis, 2009; Bonaventura et al., 2011] the real value $(E / N)_{\max }$ in the streamers becomes about 1.4 times greater than the optical data gives. According to [Kuo et al., 2009; Bonaventura et al., 2011] we assume that in the streamer head where the branching probability is highest, the value $(E / N)_{\max }$ reaches 500 - $600 \mathrm{Td}$ (it is shown by the dotted area in Fig. 1). 
111 As it follows from Fig. 1 for $E / N=500-600 \mathrm{Td}$ the fractal dimention of the branching plasma

112 channels yields:

$113 D=2.05 \pm 0.04$

114 Note that it significantly differes from $D=1.37 \pm 0.13$ used by [Pasko et al., 2000].

3. Average number of streamers in blue jets and gigantic jets

116 According to the fractal theory the full length $L$ of the streamers inside the surface having the 117 radius R can be presented by [Niemeyer et al., 1984; Wiesmann et al., 1986]:

$118 L \propto R^{D}$

119 While the number of streamers crossing this surface yields

$120 \quad N(R)=\frac{d L}{d R}=\left(\frac{R}{R_{0}}\right)^{D-1}$

121 where $R_{0}$ is the characteristic scale within which the streamer branches. The value $R_{0}$ cannot be 122 obtained from the fractal theory and thus it should be provided as an input to the model.

123 Another important result from the fractal theory describes the average inter-streamer distance $124 \Lambda$ as the function of $R$ :

125

$\Lambda(R) \propto \frac{d^{2} L}{d R^{2}} \propto\left(\frac{R}{R_{0}}\right)^{D-2}$

126

127

128

129

130

131

132

133

134

135

136

137
Equation (6) has the proportionality sign which assumes the existence of a dimensional factor, the latter is missing from Equation (6). The factor will determine dimensionality of the average inter-streamer distance.

Consider $D=2$ (see Eq. (3)) we obtain that $\Lambda(R) \approx$ const i.e. the average inter-streamer distance changes only slightly as the distance $\mathrm{R}$ increases. Moreover, using Eq. (5) one can find the total number of the streamers in the Blue Jet, $N(R)$, at the height $h_{\max }$. The latter is the maximum height where the optical steamers are the most distict, therefore they will be used in our analysis. Here $R=h_{\max }-h_{0}$ and $h_{0}$ is the height at which the BJ starts, while $R_{0}$ is the characteristic scale of the leader head at the height $h_{0}$. The latter height controls the value of the peak current (see Discussion section after Table 1.)

Knowing $N(R)$ one can find the average distance between the streamers

$R_{a v}=2 \cdot R_{s t r} / \sqrt{N(R)}$ 
Here $R_{s t r}$ is the observed radius of the streamer corona at the height $h_{\max }$. Furthermore the obtained value of $R_{a v}$ was checked against the streamer radius observed at the height $h_{\max }$.

\section{Relation between the streamer speed and reduced diameter}

141

142

143

144

145

146

147

148

149

150

151

152

153

154

155

156

157

158

159

160

161

162

163

164

165

166

In order to complete and test the fractal model we use the semi-empirical relations derived in [Raizer et al., 1998; Raizer et al., 2007]. The latter are based on studies of the laboratory streamers. The main input parameter for those relations is the voltage $\Delta U_{t}=U_{t}-U_{0}$ where $U_{t}$ and $U_{0}$ are the potential of the streamer tip and of the external electric field respectively. The semi-empirical relations provides us with:

Field at the streamer tip $E_{m} \approx 1.5 \cdot 10^{4}\left(N / N_{0}\right) \mathrm{kV} / \mathrm{m}$;

Radius of the tip $r_{m} \approx 3.3 \cdot 10^{-5} \Delta U_{t}\left(N_{0} / N\right) \mathrm{m} ; \Delta U_{t}[\mathrm{kV}]$

Speed of the positive streamer $\mathrm{v}_{s} \approx 5.3 \cdot 10^{4} \Delta U_{t} \mathrm{~m} / \mathrm{s} ; \Delta U_{t} \geq 5 \mathrm{kV}$;

If radius of the streamer tip is known, the voltage $\Delta U_{t}$ and the streamer speed could be obtained. On the other hand, knowing the streamer speed $V_{s t r}$ one can obtain $\Delta U_{t}$ and $r_{m}$. Note also that $\Delta U_{t}$ is unequivocally coupled to $E_{m}$ and $r_{m}$. For instance, for semi-spherical streamer tip $\Delta U_{t} \approx E_{m} r_{m}$

Let us present the equations in terms of the reduced streamer diameter $d_{r d}=2 r_{\mathrm{m}} \cdot\left(N / N_{0}\right)$ and reduced electric field $E_{m} \cdot\left(N_{0} / N\right)$. We exclude $\Delta U_{t}$ from the equations for $r_{\mathrm{m}}$ and $V_{s t r}$ and then obtain the following relation between the streamer speed and its reduced diameter

$$
V_{s t r}(\mathrm{~m} / \mathrm{s})=0.8 \cdot 10^{6} \cdot d_{r d}(\mathrm{~mm})
$$

The solid line in Figure 2 shows the streamer speed vs. the reduced diameter computed with Eq. (8). The two dashed lines are adapted from [Kanmae et al., 2012]. They were computed for the $E_{s}$ values 120 and $150 \mathrm{kV} / \mathrm{cm}$, and validated by comparison with the observations of the Red Sprite streamers. Figure 2 shows that our semi-empirical model is in agreement with that of [Kanmae et al., 2012].

\section{Discussion}

We begin with analysis of the GJ images [Pasko et al., 2002; Su et al., 2003]. The image from [Su et al., 2003] is revealed in Fig. 3 while two other images are not shown, just described here. Listed in the top three rows of Table 1 are the hight $h_{s t}$ where the BJ starts, and the maximum 
height $h_{\max }$ where the optical streamers are most distinct, along with the radia of the streamer coronas $R_{\text {str }}$ measured at $h_{\max }$.

Listed in Table 1 is also the relative air density at $h_{s t}$ and $h_{\max }$ altitude. It was obtained from the model of the earth atmosphere, and then used to compute the diameter of the leader head $R_{0}$. We assumed then that the diameter of the leader head $R_{0}$ is $1 \mathrm{~cm}$ at the ground, and it varies with the

172 height inversely proportional to the air density.

173

174

175

176

177

178

179

180

Such height dependence is based on the following considerations. According to the modern understanding [Bazelyan and Raizer, 1998; da Silva and Pasko, 2013] the value $\mathrm{R}_{0}$ is determined by the characteristic rate of the electron attachment to the molecular oxygen. The latter process involves either two particles $\left(e+\mathrm{O}_{2} \rightarrow \mathrm{O}^{-}+\mathrm{O}\right.$ ), or three particles $\left(\mathrm{e}+\mathrm{O}_{2}+\mathrm{O}_{2} \rightarrow \mathrm{O}_{2}{ }^{-}+\mathrm{O}_{2}\right.$ ). At high altitude where BJs occur the dominant is the dissociative electron attachment, which is two particle reaction (see [da Silva and Pasko, 2013]). Therefore we assume the linear dependence $R_{0}$ $\propto\left(N_{0} / N\right)$.

Theoretical estimates, based on the fractal model, show the total amount of the streamers $N_{\text {tot }}$ at $h_{\max }$ and the average inter-streamer distance (see Eq. (7)).

As it shown in [Milikh et al., 2016], in the streamer zone of a leader the strong inter-streamer interaction occurs when the streamer diameter is about the same as the distance between the streamers. Therefore we assume that the reduced streamer diameter is equal to the reduced interstreamer distance.

Finally using Eq. (8) we compute the streamer speed, which is in the range of $(0.3-2.0) \cdot 10^{7} \mathrm{~m} / \mathrm{s}$. Note that the experiments [Wescott et al., 2001; Pasko et al., 2002; Su et al., 2003] lack fast speed optical detectors, thus the temporal resolution of the observed images was insufficient for accurate detection of the streamer speed. As mentioned by [Pasko et al., 2002] "the estimated speed in the range $(1.9-2.2) \cdot 10^{6} \mathrm{~m} / \mathrm{s}$ is therefore a lower bound on the actual speed, which was probably higher".

\begin{tabular}{|l|c|c|}
\hline Observables & $\begin{array}{c}\text { [Pasko et al., 2002]; } \\
\text { [da Silva and Pasko, 2012] }\end{array}$ & [Su et al., 2003, fig. 2] \\
\hline Height of streamers start $h_{\text {st }}$ & $35 \mathrm{~km}$ & $37.5 \mathrm{~km}$ \\
\hline $\begin{array}{l}\text { Maximum height streamers reach } \\
h_{\max }\end{array}$ & $67 \mathrm{~km}$ & $73 \mathrm{~km}$ \\
\hline $\begin{array}{l}\text { Radius of the streamer corona } \\
R_{\text {str }} \text { at } h_{\max }\end{array}$ & $5 \mathrm{~km}$ & $5.9 \mathrm{~km}$ \\
\hline
\end{tabular}

\begin{tabular}{|l|c|c|}
\hline Estimates & [Pasko et al., 2002]; & [Su et al., 2003, fig. 2] \\
\hline $\begin{array}{l}\text { Relative air density } \\
N(h=0) / N\left(h_{s t}\right)\end{array}$ & 145 & \\
\hline
\end{tabular}




\begin{tabular}{|l|c|c|}
\hline$N(h=0) / N\left(h_{\max }\right)$ & 9,000 & 24,500 \\
\hline Total branches $N_{\text {tot }}$ at $h_{\max }$ & 22,000 & 17,400 \\
\hline $\begin{array}{l}\text { Average inter-streamer distance, } \\
R_{a v} \text { at } h_{\max }\end{array}$ & $67.3 \mathrm{~m}$ & $89.4 \mathrm{~m}$ \\
\hline Reduced streamer diameter & $7.5 \mathrm{~mm}$ & $3.7 \mathrm{~mm}$ \\
\hline Streamer speed (from Eq. (8)) & $6 \cdot 10^{6} \mathrm{~m} / \mathrm{s}$ & $3 \cdot 10^{6} \mathrm{~m} / \mathrm{s}$ \\
\hline
\end{tabular}

Table 1. Results of observations of Gigantic Blue Jets adopted from [Su et al., 2003; Pasko et al., 2002] (the three top rows). Estimates based on the fractal model (rows 4-6 from the top). Reduced streamer diameters are shown in the row second from the bottom. The streamer speed is presented in the bottom row.

Moreover the GJ current evaluations were carried out for the experimental conditions [Neubert et al, 2011]. The full GJ current has been estimated based on the model of a single streamer which reaches the altitude $\mathrm{h}_{\max }$ [Raizer et al., 2007]. The current amplitude of the streamer was multiplied then by the number of streamer channels $\mathrm{N}\left(\mathrm{h}_{\max }\right)$ at this altitude. The latter value was determined with the help of the fractal theory, Eq. (5). The full GJ current $I_{G J}$ was estimated for the leader head height $\mathrm{h}_{0}=35 \mathrm{~km}$ [Neubert et al, 2011] and for the $\mathrm{h}_{\max }$ between 60 and $70 \mathrm{~km}$. It was found that $I_{G J}$ ranges between 3 and $5 \mathrm{kA}$ which consistent with that detected by [Neubert et al, 2011].

\section{Conclusions.}

Multiple observations of Blue Jets (BJ) show that BJ emit a fan of streamers similar to a laboratory leader (streamer corona). In the exponential atmosphere those long streamers grow preferentially upward, producing a narrow cone confined by the aperture angle. In the presented paper the dendritic structure of streamers in corona discharge was treated by means of the fractal theory. The fractal dimension of the branching streamer channels was found.

Some BJ images available from the literature were analyzed and the average inter-streamer distance was estimated with the help of the fractal model. Based on our previous work we assumed that in the streamer zone of the leader the inter-streamer distance is about the same as the streamer diameter. Using this assumption and the semi-empirical model [Raizer et al., 1998] we obtained the average streamer speed and compared it with the observations.

According to [Popov, 2002] the fractal dimension of the streamer channels is determined only by the reduced electric field $(\mathrm{E} / \mathrm{N})_{\mathrm{h}}$ in the streamers head. Therefore, applied to the problem of highaltitude lightning, the presented theory predicts that the fractal dimension should stay almost constant when the streamers propagate at different altitudes and different values of air density. In 
addition, [Popov, 2002] showed that the fractal of the group of the streamer channels is not affected by the applied voltage thus it does not depend on the leader potential difference with respect to the ionosphere.

Full GJ current $I_{G J}$ was estimated for the observational conditions [Neubert et al., 2011]. It was shown that $I_{G J}$ ranges between 3 and $5 \mathrm{kA}$ which consistent with the detected values.

\section{Acknowledgments}

This work was supported by the NSF grant 1226237 .

\section{References}

Bazelyan, E. M., Raizer Y.P., 1998. Sparks Discharge. CRC Press, Florida.

Bonaventura Z., A. Bourdon, S. Celestin, V.P. Pasko, 2011. Electric field determination in streamer discharges in air at atmospheric pressure. Plasma Sources Sci. Technol., 20, 035012, doi:10.1088/0963-0252/20/3/03501.

Chou J. K., L. Y. Tasi, C. L. Kuo, Y. J. Lee, C. M. Chen, A. B. Chen, H. T. Su, R. R. Hsu, P. L. Chang, L. C. Lee, 2011. Optical emissions and behaviors of the blue starters, blue jets, and gigantic jets observed in the Taiwan transient luminous event ground campaign. J. Geophys. Res., 116, A07301, doi: 10.1029/2010JA016162.

Cummer, S. A., J. Li, F. Han, G. Lu, N. Jaugey, W. A. Lyons, T. E. Nelson, 2009. Quantification of the troposphere-to-ionosphere charge transfer in a gigantic jet. Nat. Geosci., 2, 617-620, doi: 10.1038/NGEO607.

da Silva C.L., and Pasko V.P., 2012. Simulation of leader speeds at gigantic jet altitudes. Geophys. Res. Lett. 39, L13805, doi:10.1029/2012GL052251

da Silva C.L., and Pasko V.P., 2013. Vertical structuring of gigantic jets. Geophys. Res. Lett. 40, 3315, doi:10.1002/grl.50596

Hagelaar, G.J., and L.C. Pitchford, 2005. Solving the Boltzmann equation to obtain electron transport coefficients and rate coefficients for fluid models. Plasma Sources Sci. Technol., 14, 722, doi:10.1088/0963-0252/14/4/011.

Kanmae T., H.C. Stenbaek-Nielsen, M.G. McHarg, R.K. Haaland, 2012. Diameter-speed relation of sprite streamers. J. Phys. D: Appl. Phys., 45, 275203, doi:10.1088/0022-3727/45/27/275203. 
253 Kuo, C.L., J. K. Chou, L. Y. Tsai, A. B. Chen, H. T. Su, R. R. Hsu, S. A. Cummer, H. U. Frey, 254 S. B. Mende, Y. Takahashi, L. C. Lee, 2009. Discharge processes, electric field, and electron 255 energy in ISUAL recorded gigantic jets. J. Geophys. Res., 114, A04314, 256 doi:10.1029/2008JA013791.

257 Liu, N., Pasko, V.P., 2006. Effects of photoionization on similarity properties of streamers at 258 various pressures in air. J. Phys. D:Appl. Phys. 39, 327, doi:10.1088/0022-3727/39/2/013. discharges observed above Tropical Depression Dorian. Nat. Commun., 6, 5995, doi: 10.1038/ncomms6995

262

263

264

265

266

267

268

269

270

271

272

273

274

275

276

277

278

279

280

281

282

283

284

285

286

287

Milikh, G. M., Likhanskii A.V., Shneider M. N., Raina A., George A., 2016. 2-D model of the streamer zone of a leader. J. Plasma Phys., 82, 905820102, doi:10.1017/S0022377815001452.

Naidis G.V., 2009. Positive and negative streamers in air: Velocity-diameter relation. Phys. Rev. E. 79, 057401, doi: 10.1103/PhysRevE.79.057401.

Neubert, T., O. Chanrion, E. Arnone, F. Zanotti, S. Cummer, J. Li, M. Füllekrug, S. Soula, and O. van der Velde, 2011The properties of a gigantic jet reflected in a simultaneous sprite:

Observations interpreted by a model. J. Geophys. Res., 116, A12329, doi:10.1029/2011JA016928.

Niemeyer L., L. Pietronero, H.J. Wiesmann, 1984. Fractal dimension of dielectric breakdown. Phys. Rev. Lett., 52, 1033, doi: 10.1103/PhysRevLett.52.1033.

Pasko, V.P., Inan U.S., Bell T.F., 2000. Fractal structure of sprites. Geophys. Res. Lett. 27(4), 497, doi:10.1029/1999 GL010749.

Pasko, V.P., and George J., 2002 Three-dimensional modeling of blue jets and blue starters J. Geophys. Res., 107, A12, doi: 10.1029/2002JA009473

Pasko, V. P., Stanley M. A., Matthews J. D., Inan U.S., Wood T. G., 2002. Electrical discharge from a thundercloud top to the lower ionosphere. Nature, 416, 152, doi:10.1038/416152a.

Popov N.A., 2002. Spatial structure of the branching streamer channelsin a corona discharge. Plasma Physics Reports, 28(7), 615, doi: 10.1134/1.1494061.

Raizer Yu.P., Milikh G.M, Shneider M.N., Novakovski S.V., 1998. Long streamers in the upperatmosphere above thundercloud. J. Phys. D: Appl. Phys., 31, 3255, PII: S00223727(98)94154-7.

Raizer Yu.P., Milikh G.M., Shneider M.N., 2006. On the mechanism of blue jet formation and propagation. Geophys. Res. Lett. 33, L23801, doi:10.1029/2006GL027697

Raizer Yu.P., Milikh G.M., Shneider M.N., 2007. Leader-streamers nature of Blue Jets. Journal of Atmospheric and Solar-Terrestrial Physics, 69, 925, doi:10.1016/j.jastp.2007.02.007. 
288 Satpathy, S., 1986. Dielectric breakdown in three dimensions, in: Pietronero, L., Tosatti, E.

289 (Eds.), Fractals in Physics. Elsevier, pp. 173-190.

290 Soula S., O. van der Velde, J. Montanya, P. Huet, C. Barthe, J. Bór, 2011. Gigantic jets produced by an 291 isolated tropical thunderstorm near Rerunion Island. J. Geophys. Res. 116, D19103, 292 doi:10.1029/2010JD015581.

293 Su, H. T., R. R. Hsu, A. B. Chen, Y. C. Wang, W. S. Hsiao, W. C. Lai, L. C. Lee, M. Sato, H. 294 Fukunishi, 2003. Gigantic jets between a thundercloud and the ionosphere. Nature, 423, 974, 295 doi:10.1038/nature01759.

296 Wescott, E. M., D. Sentman, D. Osborne, D. Hampton, M. Heavner, 1995. Preliminary results 297 from the Sprites 94 aircraft campaign, 2, Blue jets. Geophys. Res. Lett. 22(10), 1209, 298 doi:10.1029/95GL00583.

299 Wescott, E. M., D. Sentman, H. C. Stenbaek-Nielsen, P. Huet, M. J. Heavner, and D. R. Moudry, 300 2001. New evidence for the brightness and ionization of blue jets and blue starters. J. Geophys. 301 Res., 106 (A10), 21, 549, doi:10.1029/2000JA000429.

302 Wiesmann, H. J., L. Pietronero, 1986. Properties of Laplacian fractals for dielectric breakdown 303 in 2 and 3 dimensions, in: Pietronero, L., Tosatti, E. (Eds.), Fractals in Physics. Elsevier, pp. 304 151-172.

305

306

307

308

309

310

311

312

313

Figure Captions

Figure 1. The fractal dimension $D$ (solid line) and the power index gamma (the dashed line) versus 8 reduced electric field $\mathrm{E} / \mathrm{N}$ computed for the streamer tips. The rectangular box contains the $\mathrm{Td}$.

Figure 2. Speed versus the reduced diameter. The solid line is computed using relation (8) of our model. The two dashed lines are adapted from [Kanmae et al., 2012] where they were computed for $E_{s}=120$ and $150 \mathrm{kV} / \mathrm{cm}$, and validated by comparison with the observations of the Red Sprite streamers.

315 Figure 3. Image of the gigantic blue jet [Su et al., 2003]. The leader starts at $h_{s t}=37.5 \mathrm{~km}$; the 316 maximum altitude considered $h_{\max }=73 \mathrm{~km}$; radius of the streamer corona $R_{s t r}=5.9 \mathrm{~km}$ 317 measured at $h_{\max }$. 


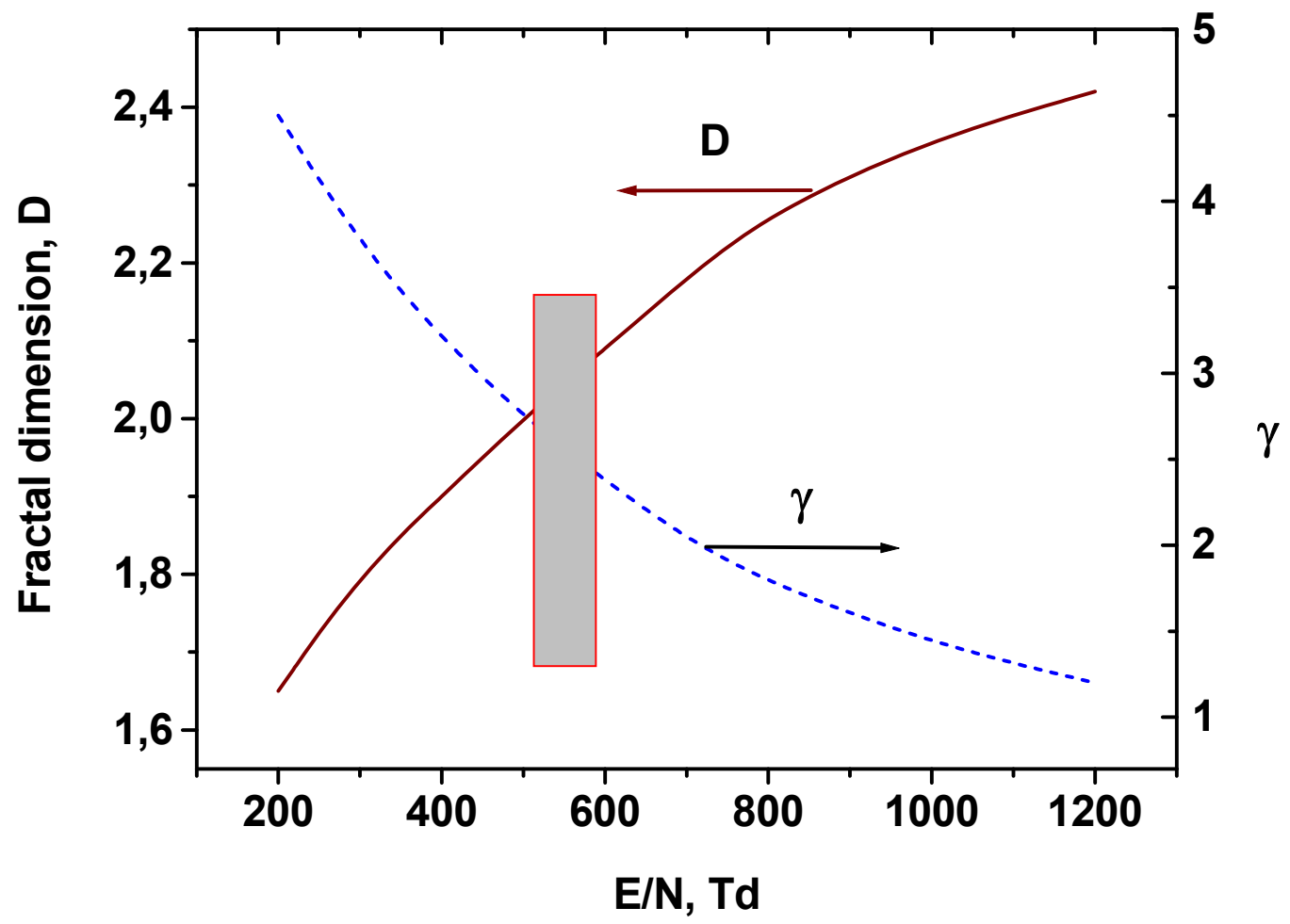

319 Figure 1. The fractal dimension D (solid line) and the power index gamma (the dashed line) 320 versus reduced electric field $\mathrm{E} / \mathrm{N}$ computed for the streamer tips. The rectangular box contains 321 the variations of the coefficient $\gamma$ and of the fractal dimension in the $E / N$ reduced field range $322 \quad 500-600 \mathrm{Td}$. 


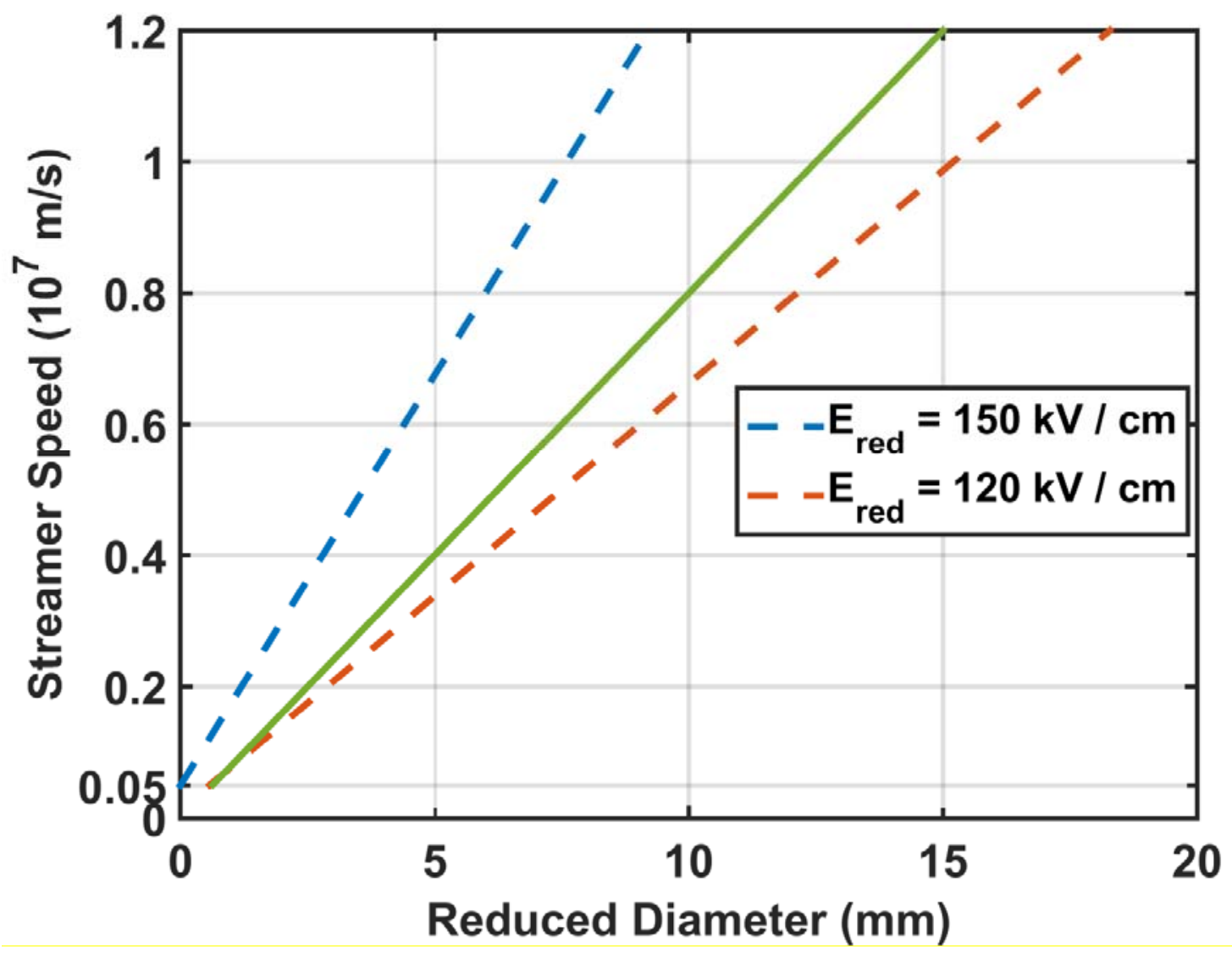

324 Figure 2. Streamer speed versus the reduced diameter. The solid line is computed using relation 325 (8) of our model. The two dashed lines were computed for $E_{\text {red }}=120$ and $150 \mathrm{kV} / \mathrm{cm}$, and 326 validated by comparison with the observations of the Red Sprite streamers [Kanmae et al., 327 2012]. 


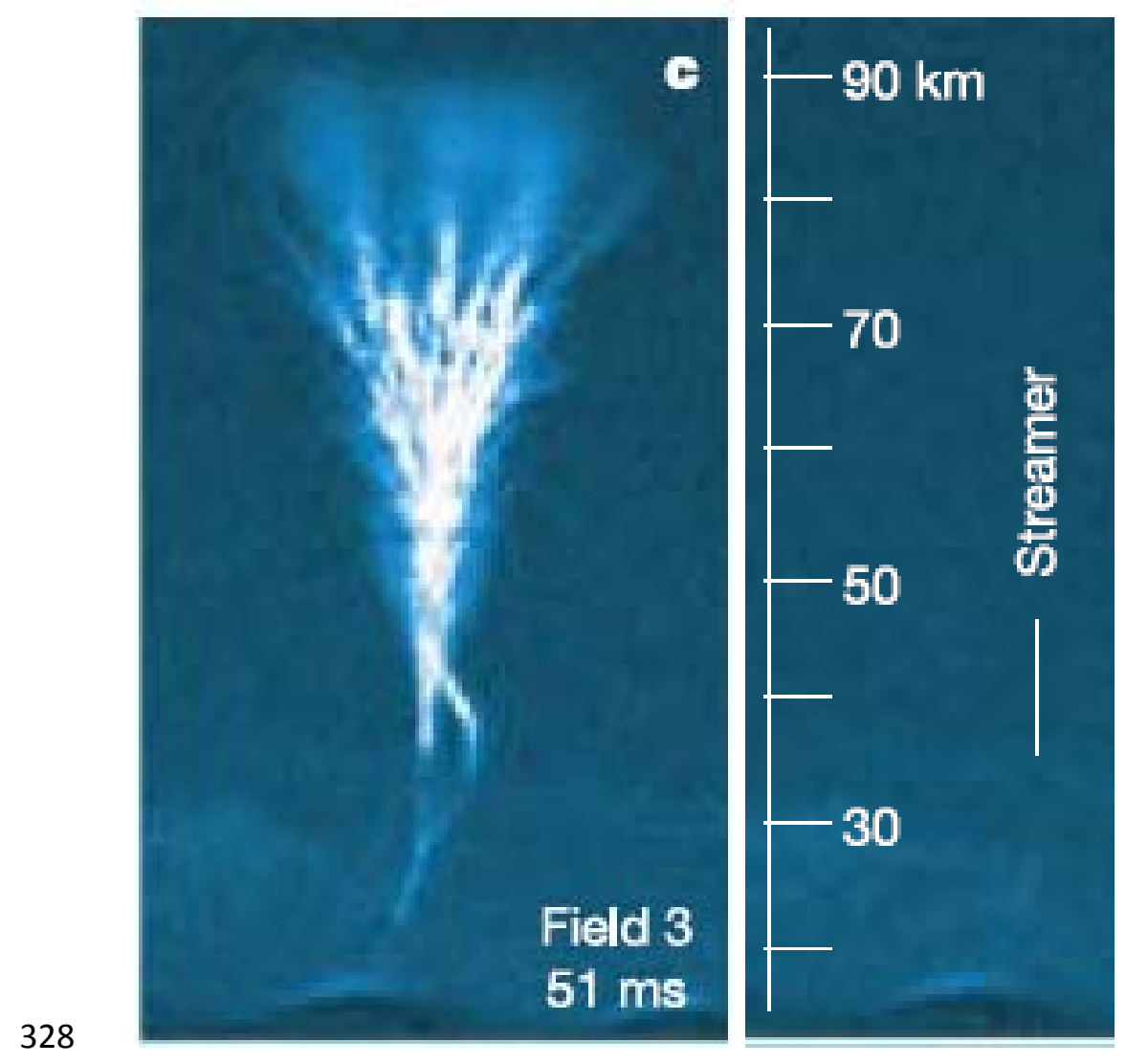

329 Figure 3. Image of the gigantic blue jet [Su et al., 2003]. The leader starts at $h_{s t}=37.5 \mathrm{~km}$; the 330 maximum altitude considered $h_{\max }=73 \mathrm{~km}$; radius of the streamer corona $R_{\text {str }}=5.9 \mathrm{~km}$ 331 measured at $h_{\max }$. 